\title{
On the Maximum Number of Independent Elements in Configurations of Points and Lines
}

\author{
Tomaž Pisanski • Thomas W. Tucker
}

Received: 10 June 2013 / Revised: 22 April 2014 / Accepted: 8 July 2014 /

Published online: 7 August 2014

(C) Springer Science+Business Media New York 2014

\begin{abstract}
We show that the upper bound for the maximum number of independent elements of a $\left(v_{r}\right)$ configuration is given by $\lfloor 2 v /(r+1)\rfloor$ and that this bound is attained for all integer values of $r$ by geometric configurations of points and lines in the Euclidean plane. This disproves a conjecture of Branko Grünbaum.
\end{abstract}

Keywords Configuration of points and lines - Unsplittable configuration . Unsplittable graph · Independent set · Levi graph · Grünbaum graph

Mathematics Subject Classification $51 \mathrm{~A} 20 \cdot 05 \mathrm{~B} 30$

\section{Introduction}

In his wonderful book Configurations of Points and Lines [4] Branko Grünbaum raises a conjecture about the maximal size of independent sets of elements in configurations of points and lines that is a motivation for this note. The question can be expressed purely in terms of graph theory. In this note we give an answer first for graphs and then translate it back to configurations.

A (combinatorial) configuration $\mathcal{C}$ of type $\left(v_{r}\right)$, or a $\left(v_{r}\right)$ configuration, is an incidence structure with sets $\mathcal{P}$ and $\mathcal{L}$ of objects called points and lines respectively, such that

\section{T. Pisanski}

Faculty of Mathematics and Physics, University of Ljubljana, Jadranska 19, 1000 Ljubljana, Slovenia e-mail: tomaz.pisanski@fmf.uni-lj.si

T. W. Tucker

Colgate University, Hamilton, NY 13346, USA

e-mail: ttucker@colgate.edu 
1. $\mathcal{P} \cap \mathcal{L}=\emptyset$.

2. $|\mathcal{P}|=|\mathcal{L}|=v$.

3. each line is incident with $r$ points,

4. each point is incident with $r$ lines,

5. two distinct points are incident with at most one common line.

A geometric $\left(v_{r}\right)$ configuration is a set of $v$ points and $v$ (straight) lines in the Euclidean plane, such that precisely $r$ of the lines pass through each of the points, and precisely $r$ of the points lie on each of the lines. It is clear that each geometric configuration determines a combinatorial configuration, while the converse is not true [4].

Given a configuration $\mathcal{C}$ of type $\left(v_{r}\right)$, its Levi graph $L(\mathcal{C})$, see [2] (also called the incidence graph), is a $r$-regular bipartite graph with vertex parts $\mathcal{P}$ and $\mathcal{L}$ and edges between $p \in \mathcal{P}$ and $\ell \in \mathcal{L}$, whenever the point $p$ lies on the line $\ell$. By condition 5 in the definition for $\mathcal{C}$, the girth of $L(\mathcal{C})$ is at least 6. Conversely, each bipartite $r$-regular graph with girth at least 6 determines a pair of mutually dual $\left(v_{r}\right)$ (combinatorial) configurations. We say that a configuration is connected whenever its Levi graph is connected.

The members of $\mathcal{P} \cup \mathcal{L}$ are called elements of the configuration. Two elements are said to be independent if they are two points not belonging to a common line, or two lines not intersecting in a point, or a point and a line where the point does not lie on the line. Independence of elements is an equivalence relation on $\mathcal{P} \cup \mathcal{L}$. In this note we are interested in the maximal size of an independent set of elements of a $\left(v_{r}\right)$ configuration.

Let $G$ be a graph and let the square of $G$, denoted $G^{2}$, be the graph with vertex set $V(G)$ and an edge between $u$ and $w$ if their distance in $G$ is $d(u, w) \leq 2$, that is there is a path of length at most 2 between $u$ and $w$ in $G$. If $L=L(\mathcal{C})$ for a configuration $\mathcal{C}$, the edges of the graph $L^{2}$ correspond to incidences of point on line, two points on the same line, and two lines through the same point. Although the square is a common graph-theoretic construction, in the context of configurations [5] we call $L^{2}$ the Grünbaum graph of $C$. In particular, two elements of the configuration $\mathcal{C}$ are independent if and only if they are independent vertices in the graph $L^{2}$. Recently it has been shown that the Grünbaum graph uniquely determines a configuration of points and lines $[1,4,5]$.

Motivated by geometric reasons, Grünbaum defined a configuration $C$ to be unsplittable if for any set $S$ of independent elements of $\mathcal{C}$ the vertex-deleted subgraph $L-S$ of the corresponding Levi graph $L=L(\mathcal{C})$ is connected.

We may generalize slightly the idea of unsplittable configurations and carry it over to graphs. Define a connected graph $G$ to be unsplittable if for any independent set $S$ from $G^{2}$ the graph $G-S$ remains connected.

\section{An Upper Bound}

Given a $\left(v_{r}\right)$ configuration $\mathcal{C}$, let $M$ be the size of a maximal independent set of elements of $\mathcal{C}$. Grünbaum conjectured an upper bound of $M \leq\lfloor v / r\rfloor+1$ (Conjecture 5.1.1, page 305 of [4]). In this section we establish instead that $M \leq 2 v /(r+1)$. Furthermore for any integer $r>1$, we construct a $\left(v_{r}\right)$ configuration with $v$ divisible 
by $r+1$ such that $M$ achieves the bound. We note that $2 v /(r+1)>v / r+1$ if and only if $v /(r+1)>r /(r-1)$. Since $r /(r-1)<2$, such a configuration is a counterexample to Grünbaum's conjecture as long as $v \neq r+1$.

We first prove a general result for graphs:

Theorem 1 Let $G$ be a regular graph on $n$ vertices of valence $r$ and let $M$ be the size of the largest independent set of $G^{2}$. Then $M \leq\lfloor n /(r+1)\rfloor$.

Proof Each of the $M$ independent vertices of $G^{2}$ is incident with $r$ vertices in $G$, in total $M r$ distinct vertices that are also different from the original $M$ vertices. Hence $M+r M \leq n$ and the result follows readily.

As a consequence we obtain the following upper bound for configurations.

Theorem 2 Let $M$ be the size of an independent set of elements of a $\left(v_{r}\right)$ configuration. Then $M \leq\lfloor 2 v /(r+1)\rfloor$.

Proof Apply the previous Theorem to the Levi graph of the configuration with $n=2 v$.

For each $r$, we show that there is a geometric $\left(v_{r}\right)$ configuration achieving the upper bound of this theorem.

Theorem 3 For each integer $r \geq 3$, there exists an integer $v$, divisible by $r+1$, and a connected geometric $\left(v_{r}\right)$ configuration with $M=2 v /(r+1)$. In particular, this configuration contradicts Grünbaum's conjecture.

Proof Given an arbitrary geometric $\left(V_{r-1}\right)$ configuration $\mathcal{C}$ in the plane with no pair of parallel lines, make $r$ copies of $\mathcal{C}$ stacked in the vertical direction. We note in passing that any geometric configuration can be moved by a projective transformation to a position so that it has no pair of parallel lines, and that for any $r \geq 3$, there exists such geometric $\left(V_{r-1}\right)$ configuration for some $V$ (alternatively, the construction is by induction on $r$ with the triangle serving as the initial case $\left(V_{2}\right)$.) Connect the corresponding points in each copy by a new vertical line (introducing $V$ vertical lines this way). For each line direction in the original $\left(V_{r-1}\right)$ configuration, add a point at infinity (introducing $V$ points in this way). The newly obtained structure $\mathcal{S}$ is a $\left(v_{r}\right)$ configuration with $v=(r+1) V$. Note that $\mathcal{S}$ has $2 v /(r+1)=2 \mathrm{~V}$ independent elements consisting of the $V$ new lines and $V$ new points at infinity. Since $\mathcal{S}$ is projectively equivalent to a plane geometric configuration of points and lines, this completes the construction. Since $V>1$ and $v=(r+1) V$, we have a contradiction to Grünbaum's conjecture.

This construction can be interpreted combinatorially beginning with a Levi graph $L$ of type $\left(V_{r-1}\right)$. Take $r$ copies of $L$ to form a disconnected Levi graph $H$ which has type $\left((r V)_{r-1}\right)$. If $p$ is a point-vertex in $G$, call the corresponding $H$ vertices $p_{1}, \ldots p_{r}$. Do the same for line vertices. Add $r$ extra line-vertices and $r$ extra point vertices. Then add edges between $p_{1}, \ldots p_{r}$ and one of the extra line-vertices and repeat for the other point vertices (with a different extra line-vertex). Repeat for linevertices joined to an extra point vertex. The result is a bipartite graph of type $v_{r}$ where $v=(r+1) V$. The $V$ extra point-vertices and $V$ extra line-vertices are all independent, so $M \geq 2 V=2 v /(r+1)$. 
The configurations given by this construction are splittable, and we know of no construction of unsplittable geometric configurations attaining the upper bound for general $r$. However, we can give one for the case $r=3$. The example is $G=G(8,3)$, the Möbius-Kantor graph, or generalized Petersen graph, with vertices $u_{i}, w_{i}$ for $0 \leq i \leq 7$ and edges $u_{i} w_{i}, u_{i} u_{i+1}, w_{i} w_{i+3}$ for all $i$ modulo 8 . The graph $G$ is bipartite with girth at least 6 , so it is the Levi graph of a configuration $\mathcal{C}$, namely the Möbius-Kantor configuration. It is easily checked that the largest independent set for $G^{2}$ (a set of vertices in $G$ whose distances apart are at least 3 ), looks like $u_{0}, u_{4}, w_{2}, w_{6}$, up to graph isomorphism. It is also easily checked that such a set does not separate $G^{2}$, so the configuration $\mathcal{C}$ is unsplittable. Since $4=2 \cdot 8 /(3+1)$, the upper bound is achieved.

Given a $\left(v_{r}\right)$ configuration achieving the upper bound, we can construct infinitely many, for the same $r$, using covering graphs [3] (viewing connected, finite graphs $G$ and $H$ as topological spaces, a covering map $p: H \rightarrow G$ is a local homeomorphism onto $G)$.

Theorem 4 Let $G$ be a regular graph on $n$ vertices of valence $r$ such that $r+1$ divides $n$ and such that the upper bound for the size $M$ of a maximal independent set in $G^{2}$ is attained: $M=n /(r+1)$. Let $H$ be a covering graph over $G$. Then the maximal set of independent vertices of $H^{2}$ also attains the upper bound.

Proof Let $p: H \rightarrow G$ be the covering map and let $S$ be a set of independent vertices in $G^{2}$ whose size $M$ achieves the upper bound. We claim that $p^{-1}(S)$ is an independent set in $H^{2}$; it clearly attains the bound since $H$ has the same valence as $G$ and the number of vertices in $H$ is a multiple of $n$. Suppose that $u, w \in p^{-1}(S)$ are dependent in $H^{2}$ so they are joined by a path $Q$ of length 1 or 2 in $H$. Then $p(Q)$ has length 1 or 2. Since $p(u), p(w)$ are in $S$ and hence independent, it must be that $p(u)=p(w)$. But then $p(Q)$ is a single edge $e$, as $G$ does not have multiple edges. This is impossible since $p^{-1}(e)$ consists of disjoint edges and cannot contain the path $Q$.

Corollary 5 Given any $r \geq 3$, there are infinitely many $\left(v_{r}\right)$ combinatorial configurations with $2 v /(r+1)$ independent elements.

Proof We take as $G$ any Levi graph like that given in Theorem 3. Then any covering graph $H$ is bipartite with at least the same girth, so such a graph is a Levi graph for some configuration.

We do not know if there are, for each $r \geq 3$, infinitely many geometric configurations attaining the bound.

In general, configurations may be unbalanced, i.e. the corresponding Levi graph may be only semi-regular. If we have $v$ black vertices, each of valence $r$ and $b$ white vertices, each of valence $k$, the corresponding incidence structure is called a $\left(v_{r}, b_{k}\right)$ configuration. In this context, we get the following upper bound.

Theorem 6 Let $M$ be the size of an independent set of elements of $a\left(v_{r}, b_{k}\right)$ configuration. Then $M \leq\lfloor(b k+v r-v-b) /(r k-1)\rfloor$. 
Proof Let $M=p+q$ be the maximal number of independent elements, where there are $p$ points and $q$ lines. Then $p r+q \leq b, q k+p \leq v$. Clearly, if we take both equalities, the maximum for $M$ will be achieved. Solving for $p$ and $q$ we get $M=(b k+v r-v-b) /(r k-1)$ and the result follows. Note that in case $b=v$ and $k=r$ we obtain the same result as Theorem 3 .

It would be interesting to find geometric realizations of the upper bound for nonbalanced configurations.

Acknowledgments The authors would like to thank Branko Grünbaum and Peter Horak for fruitful discussion. The work was supported in part by a Grant from the Picker Institute at Colgate University, the ARSS of Slovenia, Grants: P1-0294 and N1-0011: GReGAS, and by the European Science Foundation.

\section{References}

1. Adamaszek, A., Adamaszek, M.: Uniqueness of graph square roots of girth six. Electron. J. Comb. 18 \#P139 (2011)

2. Coxeter, H.S.M.: Self-dual configurations and regular graphs. Bull. Am. Math. Soc. 56, 413-455 (1950)

3. Gross, J.L., Tucker, T.W.: Topological Graph Theory. Wiley, New York (1987) and Dover, Mineola (2001)

4. Grünbaum, B.: Configurations of Points and Lines. Graduate Studies in Mathematics, vol. 103. American Mathematical Society, Providence, RI (2009)

5. Pisanski, T., Servatius, B.: Configurations from a Graphical Viewpoint. Birkhäuser Advanced Texts Basler Lehrbücher Series. Birkhäuser Boston, Boston (2013) 